\title{
Local existence and blow up of solutions to a Petrovsky equation with variable-exponent nonlinearities
}

\author{
Jorge Ferreira ${ }^{1}$ and Erhan Pişkin ${ }^{2}$ \\ ${ }^{1}$ Federal Fluminense University - Volta Redonda Campus \\ ${ }^{2}$ Dicle University
}

May 5, 2020

\begin{abstract}
In this paper, we consider a nonlinear plate (or beam) Petrovsky equation with strong damping and source terms with variable exponents. The exponents of nonlinearity $p([?])$ and $q([?])$ are given functions. By using the Banach contraction mapping principle the local existence of a weak solutions is established under suitable assumptions on the variable exponents $p$ and $p$. We also show a finite time blow up result for the solutions with negative initial energy.
\end{abstract}

\section{Hosted file}

paper.pdf available at https://authorea.com/users/303586/articles/433777-1ocal-existenceand-blow-up-of-solutions-to-a-petrovsky-equation-with-variable-exponent-nonlinearities

\section{Introduction}

Let be $\Omega$ a bounded domain in $\mathbb{R}^{n}(n \geq 1)$ with a sooth boundary $\partial \Omega$. We consider the following initial boundary value problem:

$$
\begin{array}{ll}
u_{t t}+\Delta^{2} u-\Delta u_{t}+\left|u_{t}\right|^{p(x)-2} u_{t}=|u|^{q(x)-2} u, & (x, t) \in \Omega \times(0, T) \\
u(x, 0)=u_{0}(x), u_{t}(x, 0)=u_{1}(x), & x \in \Omega \\
u(x, t)=\partial_{v} u(x, t)=0, & x \in \partial \Omega
\end{array}
$$

where $v$ is the unit outer normal to $\partial \Omega$ and the expoents $p(\cdot)$ and $q(\cdot)$ are given measurable functions on $\Omega$ satisfyning.

$2 \leq p^{-} \leq p(x) \leq p^{+} \leq p^{*}$

$2 \leq q^{-} \leq q(x) \leq q^{+} \leq q^{*}$ 
where

$$
\begin{aligned}
& p^{-}=\text {ess } \inf _{x \in \Omega} p(x), \quad p^{+}=\text {ess } \sup _{x \in \Omega} p(x) \\
& q^{-}=\text {ess } \inf _{x \in \Omega} q(x), \quad q^{+}=\text {ess } \sup _{x \in \Omega} q(x)
\end{aligned}
$$

and

$$
\begin{gathered}
p^{*}, q^{*}=\{ \\
\infty, \quad \text { if } n \leq 4 \\
\frac{2 n}{n-4}, \quad \text { if } n>4 .
\end{gathered}
$$

When $p(x)$ and $q(x)$ are constants and without strong damping $-\Delta u_{t}$, become the following the Petrovsky equation (3). Messaoudi, in (S. A. Messaoudi \& nonexistence in a, n.d.), studied the following problem

$$
\begin{aligned}
& \{ \\
& u_{t t}+\Delta^{2} u+\left|u_{t}\right|^{m-2} u_{t}=|u|^{p-2} u \quad \text { in } Q_{T}=\Omega \times(0, T) \\
& u=\partial u / \partial v=0 \quad \text { on } \Gamma_{T}=\partial \Omega \times[0, T) \\
& u(x, 0)=u_{0}(x), \quad u_{t}(x, 0)=u_{1}(x) \quad \text { in } \Omega
\end{aligned}
$$

established an existence result and showed that the solution continution continues to exist globally if $m \geq p$, and blows up in finite time if $m<p$ and the initial energy is negative. This result was later improved by Chen and Zhou in (W. Chen, n.d.). For more results related to the plate equations, we refer the reader to Lagnese (J. Lagnese, 1989), Horn and Lasiecka (1994). Lasiecka (I. Lasiecka \& plates like equaions with nonlinear dissipation on the boundary, 1989).

In recent years, some authors like [8] investigated (3) also the problem (1) with strong damping and $p$ and $q$ constants, thus,

$$
u_{t t}+\triangle^{2} u-\triangle u_{t}+\left|u_{t}\right|^{p-2} u_{t}=|u|^{q-2} u .
$$

Li et al. (missing citation) showed the existence, decay and blow up of the solutions of the problem (4) and proved global existence and blow up. In 2013, (E. Pişkin, n.d.) Pişkin and Polat showed the global existence and the decay of the solutions for (3).

A considerable effort has been devoted to the study of problem (1) in case of constant and variable-exponent nonlinearities.

In recent years, plate equations with lower order perturbation of $p$-Laplacian type in the form 


$$
u_{t t}+\triangle_{x}^{2} u-\operatorname{div}\left(\phi\left(\nabla_{x} u\right)\right)=F\left(u, u_{t}\right)
$$

where $\phi(z) \approx|s|^{(p-2)} s, p \geq 2$, and $F\left(u, u_{t}\right)$ representing additional damping and forcing terms. This attracted attention of several authors. It is a prototype for some important models in real-world applications.

In the absence of the viscoelastic term $(g=0)$ and replacing the $\vec{p}(x, t)$-Laplacian by $\Delta_{p} u=$ $\operatorname{div}\left(\left(|\nabla u|^{p-2} \nabla u\right)\right)(p=$ const $\geq 2)$, the equation

$$
u_{t t}+\triangle^{2} u-\operatorname{div}\left(\left(|\nabla u|^{p-2} \nabla u\right)\right)-\triangle u_{t}=h\left(x, u, u_{t}\right)
$$

has been extensively studied and results concerning existence, nonexistence and long-time behaviour have been established. See in this regard in (2003),(327AD).

In one-dimension, Eq. (4) without damping or forcing terms is related to the model

$$
\rho u_{t t}+\zeta u_{x x x x}+a\left(u_{x}^{2}\right)_{x}=0, \quad a>0 \text { and } \quad \zeta=\text { const }>0
$$

which describes elastoplastic-microstructure flows as discussed in (1994), (1995).

In two dimensions, with $p=4$ and weak damping, Eq.(4) corresponds to the so called model for nonlinear plates

$$
u_{t t}+\triangle^{2} u-\operatorname{div}\left[\left(|\nabla u|^{2} \nabla u\right)\right]+k u_{t}=\sigma \Delta\left(u^{2}\right)-f(u)
$$

This is indeed a limit of the Mindlin-Timoshenko plates as the shear modulus tends to infinity, as shows in (CHUESHOV, 2008). Remarkable results were obtained in (CHUESHOV, 2008), (2006), where the existence of finite-dimensional global attractors under a weak damping $k u_{t}$, instead of $-\Delta u_{t}$, was proved. Recently, (E. Pişkin, n.d.) proved the blow up of solutions for a nonlinear viscoelastic wave equations with variable exponents the following equation.

$$
u_{t t}-\Delta u+\int_{0}^{t} g(t-\tau) \Delta u(\tau) d \tau+\left|u_{t}\right|^{p(x)-2} u_{t}=|u|^{q(x)-2} u, \quad(x, t) \in \Omega \times(0, T)
$$

In the presence of the viscoelastic term $(g \neq 0)$, the problem (1) with memory was first studied in (2012).

The general decay of weak solutions $u=u(x, t)$ for a class of plate equations with memory term and lower order perturbation of $\vec{p}(x, t)$-Laplacian type. Precisely, we consider the following problem (for exemplo, see (FERREIRA et al., 2014)).

$$
u_{t t}=\operatorname{div}\left(|\nabla u|^{p} \nabla u\right)
$$

with the constant exponent of nonlinearity $p \in(1, \infty)$. During the last decades Eq.(5) was intensively studied and was casted for the role of a touchstone in the nonlinear PDEs. There is an extensive literature devoted to Eq.(5). The existence of global solution without an additional dissipation term is an still open problem. 
We also mention the very important contribution by (S. Antontsev et al., 2011), where the author proved the existence and blow up for the weak solution of a wave equation with $p(x, t)$-Laplacian and damping terms given by

$$
\begin{aligned}
& u_{t t}=\operatorname{div}\left(a(x, t)|\nabla u|^{p(x, t)-2} \nabla u+\varepsilon \nabla u_{t}\right)+b(x, t)|u|^{\sigma(x, t)-2} u+f(x, t), \\
& u(x, 0)=u_{0}(x), \quad u_{t}(x, 0)=u_{1}(x), \quad x \in \Omega, \\
& \left.u\right|_{\Gamma_{T}}=0 \quad \Gamma_{T}=\partial \Omega \times(0, T)
\end{aligned}
$$

where the coefficients $a, b, f$ and the exponents $p, \sigma$ are given measurable functions and $\varepsilon=$ const $>0$. Such equations (with variable exponents of nonlinearities) are usually referred as equations with nonstandard growth conditions.

Equations with nonstandard growth conditions occur in the mathematical modelling of various physical phenomena, e.g., the flows of electro-rheological fluids or fluids with temperature-dependent viscosity, nonlinear viscoelasticity, processes of filtration through a porous media and the image processing see (2009) and references therein.

It is to be noted here that in all papers (referring to the case $p \neq 0$ ) the viscous term $\varepsilon \Delta u_{t}$ plays a key role in the proof of local and global existence (even if $p=$ const $\neq 2$ ). The principal difficulty remains in proving an existence theorem by considering the term $-\Delta_{\vec{p}(x, t)} u$. The viscous term $\varepsilon \Delta u_{t}(\varepsilon>0)$ facilitates the proof of existence theorem.

In (1147), the authors improved the results from (2012) by establishing local and global existence, as well as the uniqueness of the weak solution $u(x, t)$ to problem (1).

Recently in (S. A. Messaoudi, 2020), the author established the decay of solutions of a damped quasilinear wave equation with variable-exponent nonlinearities.

Rivera et al. (1996) considered the following equation

$$
u_{t t}-\gamma \Delta u_{t t}+\Delta^{2} u-\int_{0}^{t} g(t-s) \Delta^{2} u(s) d s=0 \quad \text { in } \quad Q_{T}=\Omega \times(0, T),
$$

together with initial and dynamical boundary conditions and proved that the sum of the first and second energies decays exponentially (respectively polynomially) if the kernel $g$ decays exponentially (respectively polynomially). Alabau-Boussouira et al. (2008) looked into the following problem

$$
\begin{aligned}
& \{ \\
& u_{t t}+\Delta^{2} u-\int_{0}^{t} g(t-s) \Delta^{2} u(s) d s=f(u) \text { in } Q_{T}=\Omega \times(0, T) \\
& u=\partial u / \partial v=0 \quad \text { on } \Gamma_{T}=\partial \Omega \times[0, T) \\
& u(x, 0)=u_{0}(x), \quad u_{t}(x, 0)=u_{1}(x) \quad \text { in } \Omega
\end{aligned}
$$

and established exponential and polynomial decay results for sufficiently small initial data. Lin and Li in (Article Id 419717, 25 Pages, Http://dx.doi.org/10.1155, 2012), discussed

$$
u_{t t}-\gamma \Delta u_{t t}+\Delta^{2} u-\int_{0}^{t} g(t-s) \Delta^{2} u(s) d s=\operatorname{div}(C(f(\nabla u) \nabla u)) \quad \text { in } Q_{T}=\Omega \times(0, T)
$$

together with initial and dynamical boundary conditions similar to those imposed by Rivera et al. (1996), and established similar decay results. Yang in (2003), considered the problem

$$
\begin{aligned}
& \{ \\
& u_{\mathrm{tt}}+\Delta^{2} u+\lambda u_{t}=\sum_{i=1}^{n} \frac{\partial}{\partial x_{i}} \sigma_{i}\left(\frac{\partial u}{\partial x_{i}}\right) \quad \text { in } Q_{\tau}=\Omega \times(0, T) \\
& u=\partial u / \partial v=0 \quad \text { on } \Gamma_{T}=\partial \Omega \times[0, T) \\
& u(x, 0)=u_{0}(x), \quad u_{t}(x, 0)=u_{1}(x) \quad \text { in } \Omega
\end{aligned}
$$


for $\lambda \geq 0$ and $\sigma_{i}$ nonlinear functions. He proved, under some conditions on nonlinear terms and initial data, that the problem admits a global weak solution and the solution decays exponentially to zero as $t \rightarrow \infty$.

Motivated by $[8,11,16]$, we considered the local existence and blow up of solutions for nonlinear Petrovsky equation with variable exponents and strong damping.

To the best of our knowledge, this is the first work dealing with (1) subject to the with variable exponents and strong damping.

This paper is organized as follows. Before the main results, In Section 2, we recall the definitions of the variable-exponent Lebesgue spaces $L^{p(\cdot)}(\Omega)$, the Sobolev spaces $W^{1, p(\cdot)}(\Omega)$, and some of their properties. In Section 3, the statement and the proof of local existence and blow-up result for solutions with negative initial energy are given.

\section{Preliminaries}

In this part, we state some results about the variable exponent Lebesgue and Sobolev spaces $L^{p(x)}(\Omega)$ and $W^{1, p(x)}(\Omega)$ (see ????).

Let $p: \Omega \rightarrow[1, \infty]$ be a measurable function, where $\Omega$ is a domain of $R^{n}$. We define the variable exponent Lebesgue space by

$$
L^{p(x)}(\Omega)=\left\{u: \Omega \rightarrow R, u \text { is measurable and } \int_{\Omega}|u|^{p(x)} d x<\infty\right\},
$$

endowed with the Luxemburg norm

$$
\|u\|_{p(x)}=\inf \left\{\lambda>0: \int_{\Omega}\left|\frac{u}{\lambda}\right|^{p(x)} d x \leq 1\right\}
$$

$L^{p(x)}(\Omega)$ is a Banach space.

The variable exponent Sobolev space $W^{1, p(x)}(\Omega)$ is defined by

$$
W^{1, p(x)}(\Omega)=\left\{u \in L^{p(x)}(\Omega): \nabla u \text { exists and }|\nabla u| \in L^{p(x)}(\Omega)\right\} .
$$

Variable exponent Sobolev space is a Banach space with respect to the norm

$$
\|u\|_{1, p(x)}=\|u\|_{p(x)}+\|\nabla u\|_{p(x)} .
$$

The space $W_{0}^{1, p(x)}(\Omega)$ is defined as the closure of $C_{0}^{\infty}(\Omega)$ in $W^{1, p(x)}(\Omega)$ with respect to the norm $\|u\|_{1, p(x)}$. For $u \in W_{0}^{1, p(x)}(\Omega)$, we can define an equivalent norm

$$
\|u\|_{1, p(x)}=\|\nabla u\|_{p(x)}
$$


Let the variable exponent $p($.$) satisfy the log-Hölder continuity condition:$

$$
|p(x)-p(y)| \leq \frac{A}{\log \frac{1}{|x-y|}}, \text { for all } x, y \in \Omega \text { with }|x-y|<\delta,
$$

where $A>0$ and $0<\delta<1$.

(Poincare inequality) Let $\Omega$ be a bounded domain of $R^{n}$ and $p($.$) satisfies log-Hölder condition, then$

$$
\|u\|_{p(x)} \leq c\|\nabla u\|_{p(x)}, \text { for all } u \in W_{0}^{1, p(x)}(\Omega),
$$

where $c=c\left(p^{-}, p^{+},|\Omega|\right)>0$.

Let $p(.) \in C(\bar{\Omega})$ and $q: \Omega \rightarrow[1, \infty)$ be a measurable function and satisfy

$$
\underset{x \in \bar{\Omega}}{e s \operatorname{sinf}}\left(p^{*}(x)-q(x)\right)>0 .
$$

Then the Sobolev embedding $W_{0}^{1, p(x)}(\Omega) \hookrightarrow L^{q(x)}(\Omega)$ is continuous and compact. Where

$$
p^{*}(x)=\{
$$

$\frac{n p^{-}}{n-p^{-}}$, if $p^{-}<n$

$\infty$, if $p^{-} \geq n$.

We denote by $c$ various positive constants which may be different at different occurrences. Also, throughout this paper, we use the embedding

$$
H_{0}^{2}(\Omega) \hookrightarrow H_{0}^{1}(\Omega) \hookrightarrow L^{p}(\Omega)
$$

which implies

$$
\|u\|_{p} \leq c\|\nabla u\| \leq c\|\triangle u\|,
$$

where $2 \leq p<\infty \quad(n=1,2), 2 \leq p \leq \frac{2 n}{n-2} \quad(n \geq 3)$.

\section{Local existence}

In this part, the aim is to prove the local existence result for (1). Firstly, we state the following lemma which can be obtained by exploiting the Feado-Galerkin method and using the similar arguments as in ??.

Suppose that the exponent $p($.$) satisfies (2),(7)$ and initial data $u_{0} \in H_{0}^{2}(\Omega), u_{1} \in L^{2}(\Omega)$, then there exists a unique local solution $u$ of 


$$
\begin{array}{cll}
u_{t t}+\triangle^{2} u-\triangle u_{t}+\left|u_{t}\right|^{p(x)-2} u_{t}=f(t, x), & (x, t) \in \Omega \times(0, T), & \\
u(x, 0)=u_{0}(x), u_{t}(x, 0)=u_{1}(x), & x \in \Omega, & \\
u(x, t)=\partial_{v} u(x, t)=0, & x \in \partial \Omega, & \\
u \in L^{\infty}\left((0, T), H_{0}^{2}(\Omega)\right), & u_{t} \in L^{\infty}\left((0, T), L^{2}(\Omega)\right) \cap L^{p(.)}(\Omega \times(0, T)),
\end{array}
$$

where $f \in L^{2}(\Omega \times(0, T))$.

Suppose that $p($.$) satisfies (2), (7) and q($.$) satisfies (??) and$

$$
2 \leq p^{-} \leq p(x) \leq p^{+} \leq \frac{2(n-2)}{n-4} \quad(n>4) .
$$

Assume further that $u_{0} \in H_{0}^{2}(\Omega), u_{1} \in L^{2}(\Omega)$. Then the problem (2) has a unique local solution

$$
u \in L^{\infty}\left((0, T), H_{0}^{2}(\Omega)\right), \quad u_{t} \in L^{\infty}\left((0, T), L^{2}(\Omega)\right) \cap L^{p(.)}(\Omega \times(0, T))
$$

Existence: Let $v \in L^{\infty}\left((0, T), H_{0}^{1}(\Omega)\right)$ and $f(v)=|v|^{q(x)-2} v$. We have

$$
\begin{aligned}
\|f(v)\|^{2} & =\int_{\Omega}|v|^{2(q(x)-1)} d x \\
& \leq \int_{\Omega}|v|^{2\left(q^{-}-1\right)} d x+\int_{\Omega}|v|^{2\left(q^{+}-1\right)} d x<\infty,
\end{aligned}
$$

since

$$
2\left(q^{-}-1\right) \leq 2\left(q^{+}-1\right) \leq \frac{2 n}{n-2} .
$$

Thus, for each $v \in L^{\infty}\left((0, T), H_{0}^{1}(\Omega)\right)$, there exists a unique

$$
u \in L^{\infty}\left((0, T), H_{0}^{2}(\Omega)\right), \quad u_{t} \in L^{\infty}\left((0, T), L^{2}(\Omega)\right) \cap L^{p(.)}(\Omega \times(0, T)),
$$

satisfying the following problem

$$
\begin{array}{lc}
u_{t t}+\triangle^{2} u-\triangle u_{t}+\left|u_{t}\right|^{p(x)-2} u_{t}=f(v), & (x, t) \in \Omega \times(0, T), \\
u(x, 0)=u_{0}(x), u_{t}(x, 0)=u_{1}(x), & x \in \Omega, \\
u(x, t)=\partial_{v} u(x, t)=0, & x \in \partial \Omega .
\end{array}
$$

(9)

Define the following space

$$
X_{T}:=\left\{w: w \in L^{\infty}\left((0, T) ; H_{0}^{2}(\Omega)\right), w \in L^{\infty}\left((0, T) ; L^{2}(\Omega)\right)\right\} .
$$


$X_{T}$ is Banach space with respect to the norm

$$
\|w\|_{X_{T}}=\|w\|_{L^{\infty}\left((0, T) ; H_{0}^{2}(\Omega)\right)}+\|w\|_{L^{\infty}\left((0, T) ; L^{2}(\Omega)\right)} .
$$

We define the nonlinear mapping $S$ in the following way. For $v \in X_{T}, u=S v$ is the unique solution (9). We shall show that there exist $T>0$, such that

(i) $S: X_{T} \rightarrow X_{T}$

(ii) $S$ is a contraction mapping in $X_{T}$.

To show (i), multiplying (9) by $u_{t}$ and integrating over $\Omega \times(0, t)$, we obtain

$$
\begin{aligned}
& \frac{1}{2}\left\|u_{t}\right\|^{2}+\frac{1}{2}\|\Delta u\|^{2}+\int_{0}^{t}\left\|\nabla u_{\tau}\right\|^{2} d \tau+\int_{0}^{t} \int_{\Omega}\left|u_{\tau}\right|^{p(x)} d x d \tau \\
= & \frac{1}{2}\left\|u_{1}\right\|^{2}+\frac{1}{2}\left\|\Delta u_{0}\right\|^{2}+\int_{0}^{t} \int_{\Omega}|v|^{q(x)-2} v u_{\tau} d x d \tau .
\end{aligned}
$$

By the Young's and Sobolev-Poincare's inequalities, we get

$$
\begin{aligned}
\int_{\Omega}|v|^{q(x)-2} v u_{\tau} d x & \leq \frac{\delta}{4} \int_{\Omega} u_{t}^{2} d x+\frac{1}{\delta} \int_{\Omega}|v|^{2 q(x)-2} d x \\
& \leq \frac{\delta}{4}\left\|u_{t}\right\|^{2}+\frac{1}{\delta}\left[\int_{\Omega}|v|^{2\left(q^{-}-1\right)} d x+\int_{\Omega}|v|^{2\left(q^{+}-1\right)} d x\right] \\
& \leq \frac{\delta}{4}\left\|u_{t}\right\|^{2}+\frac{c}{\delta}\left(\|\triangle v\|^{2\left(q^{-}-1\right)}+\|\Delta v\|^{2\left(q^{+}-1\right)}\right) .
\end{aligned}
$$

Thus, by (10) and (11), we have

$$
\begin{aligned}
& \frac{1}{2}\left\|u_{t}\right\|^{2}+\frac{1}{2}\|\Delta u\|^{2}+\int_{0}^{t}\left\|\nabla u_{\tau}\right\|^{2} d \tau+\int_{0}^{t} \int_{\Omega}\left|u_{\tau}\right|^{p(x)} d x d \tau \\
\leq & \frac{1}{2}\left\|u_{1}\right\|^{2}+\frac{1}{2}\left\|\Delta u_{0}\right\|^{2}+\frac{\delta}{4} \int_{0}^{t}\left\|u_{t}\right\|^{2} d \tau \\
& +\frac{c}{\delta} \int_{0}^{t}\left(\|\Delta v\|^{2\left(q^{-}-1\right)}+\|\Delta v\|^{2\left(q^{+}-1\right)}\right) d \tau,
\end{aligned}
$$

which implies that

$$
\begin{aligned}
\sup _{t \in(0, T)}\left[\left\|u_{t}\right\|^{2}+\|\Delta u\|^{2}\right] \leq & \left\|u_{1}\right\|^{2}+\left\|\Delta u_{0}\right\|^{2}+\frac{\delta T}{2} \sup _{t \in(0, T)}\left\|u_{t}\right\|^{2} \\
& +\frac{c T}{\delta}\left[\|v\|_{X_{T}}^{2\left(q^{-}-1\right)}+\|v\|_{X_{T}}^{2\left(q^{+}-1\right)}\right] .
\end{aligned}
$$


By taking $\frac{\delta T}{2} \leq 1$, we have

$$
\|u\|_{X_{T}}^{2} \leq \lambda+\frac{c T}{\delta}\left[\|v\|_{X_{T}}^{2\left(q^{-}-1\right)}+\|v\|_{X_{T}}^{2\left(q^{+}-1\right)}\right]
$$

where $\lambda=\left\|u_{1}\right\|^{2}+\left\|\Delta u_{0}\right\|^{2}$. At this point we choose $M$ large enough, such that $\|v\|_{X_{T}} \leq M$. Then

$$
\|u\|_{X_{T}}^{2} \leq \lambda+\frac{c T}{\delta} M^{2\left(q^{+}-1\right)} \leq M^{2}
$$

if $\lambda<M^{2}$ and $T \leq T_{0}<\frac{\delta\left(M^{2}-\lambda\right)}{c M^{2\left(q^{+}-1\right)}}$. Thus we have $S: X_{T} \rightarrow X_{T}$.

Next, we show $S$ is a contraction mapping in $X_{T}$. For this purpose, we let $u_{1}=S v_{1}$ and $u_{2}=S v_{2}$, then $u=u_{1}-u_{2}$ satisfies

$$
\begin{aligned}
& u_{t t}+\triangle^{2} u-\triangle u_{t}+\left[\left|u_{1 t}\right|^{p(x)-2} u_{1 t}-\left|u_{2 t}\right|^{p(x)-2} u_{2 t}\right]=\left|v_{1}\right|^{q(x)-2} v_{1}-\left|v_{2}\right|^{q(x)-2} v_{2}, \\
& u(x, 0)=u_{0}(x), u_{t}(x, 0)=u_{1}(x), \\
& u(x, t)=\partial_{v} u(x, t)=0, \\
& x \in \partial \Omega .
\end{aligned}
$$

by $u_{t}=u_{1 t}-u_{2 t}$ and integrating over $\Omega \times(0, t)$, we obtain

$$
\begin{aligned}
& \frac{1}{2}\left\|u_{t}\right\|^{2}+\frac{1}{2}\|\Delta u\|^{2}+\int_{0}^{t}\left\|\nabla u_{\tau}\right\|^{2} d \tau \\
& +\int_{0}^{t} \int_{\Omega}\left[\left|u_{1 t}\right|^{p(x)-2} u_{1 t}-\left|u_{2 t}\right|^{p(x)-2} u_{2 t}\right]\left(u_{1 t}-u_{2 t}\right) d x d \tau \\
& \leq \frac{1}{2}\left\|u_{1}\right\|^{2}+\frac{1}{2}\left\|\Delta u_{0}\right\|^{2}+\int_{0}^{t} \int_{\Omega}\left(f\left(v_{1}\right)-f\left(v_{2}\right)\right) u_{t} d x d \tau .
\end{aligned}
$$

Since $\left[\left|u_{1 t}\right|^{p(x)-2} u_{1 t}-\left|u_{2 t}\right|^{p(x)-2} u_{2 t}\right]\left(u_{1 t}-u_{2 t}\right) \geq 0$ then (13) yields

$$
\begin{aligned}
& \frac{1}{2}\left\|u_{t}\right\|^{2}+\frac{1}{2}\|\triangle u\|^{2}+\int_{0}^{t}\left\|\nabla u_{\tau}\right\|^{2} d \tau \\
\leq & \frac{1}{2}\left\|u_{1}\right\|^{2}+\frac{1}{2}\left\|\Delta u_{0}\right\|^{2}+\int_{0}^{t} \int_{\Omega}\left(f\left(v_{1}\right)-f\left(v_{2}\right)\right) u_{t} d x d \tau .
\end{aligned}
$$

We estimate the last term on the right-hand side of (14) as follows

$$
\int_{\Omega}\left|f\left(v_{1}\right)-f\left(v_{2}\right)\right|\left|u_{t}\right| d x=\int_{\Omega}\left|f^{\prime}(\xi)\right||v|\left|u_{t}\right| d x
$$

where $v=v_{1}-v_{2}$ and $\xi=\alpha v_{1}+(1-\alpha) v_{2}, \quad 0 \leq \alpha \leq 1$. Thanks to Young's inequality and since $f(v)=$ $|v|^{q(x)-2} v$, we obtain 


$$
\begin{aligned}
\int_{\Omega}\left|f\left(v_{1}\right)-f\left(v_{2}\right)\right|\left|u_{t}\right| d x \leq & \frac{\delta}{2} \int_{\Omega}\left|u_{t}\right|^{2} d x+\frac{1}{2 \delta} \int_{\Omega}\left|f^{\prime}(\xi)\right|^{2}|v|^{2} d x \\
\leq & \frac{\delta}{2}\left\|u_{t}\right\|^{2}+\frac{\left(p_{2}-1\right)^{2}}{2 \delta} \int_{\Omega}\left|\alpha v_{1}+(1-\alpha) v_{2}\right|^{2(q(x)-2)}|v|^{2} d x \\
\leq & \frac{\delta}{2}\left\|u_{t}\right\|^{2}+c\left(\int_{\Omega}|v|^{\frac{2 n}{n-2}} d x\right)^{\frac{n-2}{2}}\left(\int_{\Omega}\left|\alpha v_{1}+(1-\alpha) v_{2}\right|^{n(q(x)-2)} d x\right)^{\frac{2}{n}} \\
\leq & \frac{\delta}{2}\left\|u_{t}\right\|^{2}+c\left(\int_{\Omega}|v|^{\frac{2 n}{n-2}} d x\right)^{\frac{n-2}{2}}\left[\left(\int_{\Omega}\left|\alpha v_{1}+(1-\alpha) v_{2}\right|^{n\left(q^{+}-2\right)} d x\right)^{\frac{2}{n}}\right. \\
& \left.+\left(\int_{\Omega}\left|\alpha v_{1}+(1-\alpha) v_{2}\right|^{n\left(q^{-}-2\right)} d x\right)^{\frac{2}{n}}\right]
\end{aligned}
$$

Since $2 \leq p^{-} \leq p(x) \leq p^{+} \leq \frac{2(n-2)}{n-4} \quad(n>4)$, we get

$$
\begin{aligned}
\int_{\Omega}\left|f\left(v_{1}\right)-f\left(v_{2}\right)\right|\left|u_{t}\right| d x \leq & \frac{\delta}{2}\left\|u_{t}\right\|^{2}+c\|\triangle v\|^{2} \cdot\left[\left\|\triangle v_{1}\right\|^{2\left(p^{+}-2\right)}+\left\|\triangle v_{1}\right\|^{2\left(p^{-}-2\right)}\right. \\
& \left.+\left\|\triangle v_{2}\right\|^{2\left(p^{+}-2\right)}+\left\|\triangle v_{2}\right\|^{2\left(p^{-}-2\right)}\right] \\
\leq & \frac{\delta}{2}\left\|u_{t}\right\|^{2}+4 c M^{2\left(p^{+}-2\right)}\|\triangle v\|^{2} .
\end{aligned}
$$

By the combining (14) and (16), we obtain

$$
\frac{1}{2}\|u\|_{X_{T}}^{2} \leq \frac{\delta}{2} T_{0}\|u\|_{X_{T}}^{2}+4 c M^{2\left(p^{+}-2\right)} T_{0}\|v\|_{X_{T}}^{2} .
$$

By choosing $\delta$ small enough, we have

$$
\|u\|_{X_{T}}^{2} \leq 8 c M^{2\left(p^{+}-2\right)} T_{0}\|v\|_{X_{T}}^{2} .
$$

Now, we choose $T_{0}$ sufficent enough so that

$$
0<8 c M^{2\left(p^{+}-2\right)} T_{0}<1 .
$$

Thus, the map $S$ is contraction. The Banach fixed point theorem implies the existence of a unique $u \in X_{T}$ satisfying $S(u)=u$. Obviously, it is a solution of (1).

Uniqueness: Suppose that (1) have two solutions $u$ and $v$. Then $w=u-v$ satisfies

$$
\begin{array}{lc}
w_{t t}+\triangle^{2} w-\triangle w_{t}+\left|u_{t}\right|^{p(x)-2} u_{t}-\left|v_{t}\right|^{p(x)-2} v_{t}=|u|^{q(x)-2} u-|v|^{q(x)-2} v, & (x, t) \in \Omega \times(0, T), \\
w(x, 0)=0, w_{t}(x, 0)=0, & x \in \Omega, \\
w(x, t)=\partial_{v} w(x, t)=0, & x \in \partial \Omega,
\end{array}
$$

Multiplying by $w_{t}$ and integrate over $\Omega \times(0, t)$, we get 


$$
\begin{aligned}
& \frac{1}{2}\left\|w_{t}\right\|^{2}+\frac{1}{2}\|\Delta w\|^{2}+\int_{0}^{t}\left\|\nabla w_{\tau}\right\|^{2} d \tau \\
& +\int_{0}^{t} \int_{\Omega}\left(\left|u_{t}\right|^{p(x)-2} u_{t}-\left|v_{t}\right|^{p(x)-2} v_{t}\right) w_{t} d x d \tau \\
& =\int_{0}^{t} \int_{\Omega}\left(|u|^{q(x)-2} u-|v|^{q(x)-2} v\right) w_{t} d x d \tau .
\end{aligned}
$$

Similarly (15), we have

$$
\left\|w_{t}\right\|^{2}+\|\Delta w\|^{2} \leq c \int_{0}^{t} \int_{\Omega}\left(\left|w_{t}(\tau)\right|^{2}+|\triangle w(\tau)|^{2}\right) d x d \tau .
$$

Thanks to Gronwall inequality, we get

$$
\left\|w_{t}\right\|^{2}+\|\triangle w\|^{2}=0
$$

Thus $w=0$. The proof is completed.

\section{Blow up of solutions}

In this part, we are going to consider the blow up of the solution for problem (1). Firstly, we give following lemma.

(S. A. Messaoudi, n.d.). If $q: \Omega \rightarrow[1, \infty)$ is a measurable function and

$2 \leq q^{-} \leq q(x) \leq q^{+}<\infty ; \quad n \leq, 4$ $2 \leq q^{-} \leq q(x) \leq q^{+}<\frac{2 n}{n-2} ; n>4$

(17)holds. Then, we have following inequalities:

i)

$$
\rho_{q(.)}^{\frac{s}{q-}}(u) \leq c\left(\|\triangle u\|^{2}+\rho_{q(.)}(u)\right)
$$

ii)

$$
\|u\|_{q^{-}}^{s} \leq c\left(\|\triangle u\|^{2}+\|u\|_{q^{-}}^{q^{-}}\right)
$$


iii)

$$
\rho_{q(.)}^{\frac{s}{q^{-}}}(u) \leq c\left(|H(t)|+\left\|u_{t}\right\|^{2}+\rho_{q(.)}(u)\right)
$$

iv)

$$
\|u\|_{q^{-}}^{s} \leq c\left(|H(t)|+\left\|u_{t}\right\|^{2}+\|u\|_{q^{-}}^{q^{-}}\right)
$$

v)

$$
c\|u\|_{q^{-}}^{q^{-}} \leq \rho_{q(.)}(u)
$$

for any $u \in H_{0}^{2}(\Omega)$ and $2 \leq s \leq q^{-}$. Where $c>1$ a positive constant and $H(t)=-E(t)$.

Now, we state and prove our blow up result.

Letr the assumptions of Theorem 4, and the initial energy $E(0)<0$ hold. Then the solution (1) blows up in finite time $T^{*}$, and

$$
T^{*} \leq \frac{1-\sigma}{\xi \sigma \Psi^{\frac{\sigma}{1-\sigma}}(0)}
$$

where $\Psi(t)$ and $\sigma$ are given in (26) and (27) respectively.

Multiplying $u_{t}$ on two sides of the problem (1), and integrating by part, we have

$$
\begin{gathered}
\frac{d}{d t}\left[\frac{1}{2}\left\|u_{t}\right\|^{2}+\frac{1}{2}\|\Delta u\|^{2}-\int_{\Omega} \frac{1}{q(x)}|u|^{q(x)} d x\right]=-\int_{\Omega} \frac{1}{p(x)}\left|u_{t}\right|^{p(x)} d x-\left\|\nabla u_{t}\right\|^{2}, \\
E^{\prime}(t)=-\int_{\Omega} \frac{1}{p(x)}\left|u_{t}\right|^{p(x)} d x,
\end{gathered}
$$

where

$$
E(t)=\frac{1}{2}\left\|u_{t}\right\|^{2}+\frac{1}{2}\|\Delta u\|^{2}-\int_{\Omega} \frac{1}{q(x)}|u|^{q(x)} d x
$$


Set

$$
H(t)=-E(t)
$$

then $E(0)<0$ and $(23)$ gives $H(t) \geq H(0)>0$. Also, by the definition $H(t)$, we have

$$
\begin{aligned}
H(t) & =-\frac{1}{2}\left\|u_{t}\right\|^{2}-\frac{1}{2}\|\triangle u\|^{2}+\int_{\Omega} \frac{1}{q(x)}|u|^{q(x)} d x \\
& \leq \int_{\Omega} \frac{1}{q(x)}|u|^{q(x)} d x \\
& \leq \frac{1}{q^{-}} \rho_{q(.)}(u) .
\end{aligned}
$$

Define

$$
\Psi(t)=H^{1-\sigma}(t)+\varepsilon \int_{\Omega} u u_{t} d x+\frac{\varepsilon}{2}\|\nabla u\|^{2},
$$

where $\varepsilon$ small to be chosen later and

$$
0<\sigma \leq \min \left\{\frac{q^{-}-p^{+}}{\left(p^{+}-1\right) q^{-}}, \frac{q^{-}-2}{2 q^{-}}\right\} .
$$

Differentiating $\Psi(t)$ with respect to $t$, and using Eq. (1), we have

$$
\begin{aligned}
\Psi^{\prime}(t)= & (1-\sigma) H^{-\sigma}(t) H^{\prime}(t)+\varepsilon \int_{\Omega}\left(u_{t}^{2}+u u_{t t}\right) d x+\varepsilon \int_{\Omega} \nabla u \nabla u_{t} d x \\
= & (1-\sigma) H^{-\sigma}(t) H^{\prime}(t)+\varepsilon\left\|u_{t}\right\|^{2}-\varepsilon\|\triangle u\|^{2} \\
& +\varepsilon \int_{\Omega}|u|^{q(.)} d x-\varepsilon \int_{\Omega} u u_{t}\left|u_{t}\right|^{p(.)-2} d x .
\end{aligned}
$$

By using the definition of the $H(t)$, it follows that

$$
\begin{aligned}
-\varepsilon q^{-}(1-\xi) H(t)= & \frac{\varepsilon q^{-}(1-\xi)}{2}\left\|u_{t}\right\|^{2}+\frac{\varepsilon q^{-}(1-\xi)}{2}\|\Delta u\|^{2} \\
& -\varepsilon q^{-}(1-\xi) \int_{\Omega} \frac{1}{q(x)}|u|^{q(.)} d x,
\end{aligned}
$$

where $0<\xi<1$. 
Adding and subtracting (29) into (28), we obtain

$$
\begin{aligned}
\Psi^{\prime}(t) \geq & (1-\sigma) H^{-\sigma}(t) H^{\prime}(t)+\varepsilon q^{-}(1-\xi) H(t) \\
& +\varepsilon\left(\frac{q^{-}(1-\xi)}{2}+1\right)\left\|u_{t}\right\|^{2}+\varepsilon\left(\frac{q^{-}(1-\xi)}{2}-1\right)\|\Delta u\|^{2} \\
& +\varepsilon \xi \int_{\Omega}|u|^{q(.)} d x-\varepsilon \int_{\Omega} u u_{t}\left|u_{t}\right|^{p(.)-2} d x .
\end{aligned}
$$

Then, for $\xi$ small enough, we get

$$
\begin{aligned}
\Psi^{\prime}(t) \geq & \varepsilon \beta\left[H(t)+\left\|u_{t}\right\|^{2}+\|\Delta u\|^{2}+\rho_{q(.)}(u)\right] \\
& +(1-\sigma) H^{-\sigma}(t) H^{\prime}(t)-\varepsilon \int_{\Omega} u u_{t}\left|u_{t}\right|^{p(.)-2} d x
\end{aligned}
$$

where

$$
\beta=\min \left\{q^{-}(1-\xi), \xi, \frac{q^{-}(1-\xi)}{2}-1, \frac{q^{-}(1-\xi)}{2}+1\right\}>0
$$

and

$$
\rho_{q(.)}(u)=\int_{\Omega}|u|^{q(\cdot)} d x .
$$

In order to estimate the last term in (31), we make use of the following Young inequality

$$
X Y \leq \frac{\delta^{k} X^{k}}{k}+\frac{\delta^{-l} Y^{l}}{l}
$$

where $X, Y \geq 0, \delta>0, k, l \in R^{+}$such that $\frac{1}{k}+\frac{1}{l}=1$. Consequently, applying the previous we have

$$
\begin{aligned}
\int_{\Omega} u\left|u_{t}\right|^{p(.)-1} d x & \leq \int_{\Omega} \frac{1}{p(x)} \delta^{p(x)}|u|^{p(x)} d x+\int_{\Omega} \frac{p(x)-1}{p(x)} \delta^{-\frac{p(x)}{p(x)-1}}\left|u_{t}\right|^{p(x)} d x \\
& \leq \frac{1}{p^{-}} \int_{\Omega} \delta^{p(x)}|u|^{p(x)} d x+\frac{p^{+}-1}{p^{+}} \int_{\Omega} \delta^{-\frac{p(x)}{p(x)-1}}\left|u_{t}\right|^{p(x)} d x,
\end{aligned}
$$

where $\delta$ is constant depending on the time $t$ and specified later. Inserting estimate (32) into (31), we get

$$
\begin{aligned}
\Psi^{\prime}(t) \geq & \varepsilon \beta\left[H(t)+\left\|u_{t}\right\|^{2}+\|\Delta u\|^{2}+\rho_{q(.)}(u)\right] \\
& +(1-\sigma) H^{-\sigma}(t) H^{\prime}(t) \\
& -\varepsilon \frac{1}{p^{-}} \int_{\Omega} \delta^{p(x)}|u|^{p(x)} d x-\varepsilon \frac{p^{+}-1}{p^{+}} \int_{\Omega} \delta^{-\frac{p(x)}{p(x)-1}}\left|u_{t}\right|^{p(x)} d x
\end{aligned}
$$


Let us choose $\delta$ so that

$$
\delta^{-\frac{p(x)}{p(x)-1}}=k_{1} H^{-\sigma}(t),
$$

where $k_{1}, k_{2}>0$ are specified later, we obtain

$$
\begin{aligned}
\Psi^{\prime}(t) \geq & \varepsilon \beta\left[H(t)+\left\|u_{t}\right\|^{2}+\|\triangle u\|^{2}+\rho_{q(.)}(u)\right] \\
& +(1-\sigma) H^{-\sigma}(t) H^{\prime}(t)-\varepsilon k_{2} H^{-\sigma}(t) H^{\prime}(t) \\
& -\varepsilon \frac{1}{p^{-}} \int_{\Omega} k_{1}^{1-p(x)} H^{\sigma(p(x)-1)}(t)|u|^{p(x)} d x-\varepsilon \frac{p^{+}-1}{p^{+}} \int_{\Omega} k_{1} H^{-\sigma}(t)\left|u_{t}\right|^{p(x)} d x \\
\geq & \varepsilon \beta\left[H(t)+\left\|u_{t}\right\|^{2}+\|\triangle u\|^{2}+\rho_{q(.)}(u)\right] \\
& +\left(1-\sigma-\varepsilon k_{2}\right) H^{-\sigma}(t) H^{\prime}(t) \\
& -\varepsilon \frac{k_{1}^{1-p^{-}}}{p^{-}} H^{\sigma\left(p^{+}-1\right)}(t) \int_{\Omega}|u|^{p(x)} d x-\varepsilon\left(\frac{p^{+}-1}{p^{+}}\right) k_{1} H^{-\sigma}(t) \int_{\Omega}\left|u_{t}\right|^{p(x)} d x \\
\geq & \varepsilon \beta\left[H(t)+\left\|u_{t}\right\|^{2}+\|\Delta u\|^{2}+\rho_{q(.)}(u)\right] \\
& +\left[\left(1-\sigma-\varepsilon k_{2}\right)-\varepsilon\left(\frac{p^{+}-1}{p^{+}}\right) k_{1}\right] H^{-\sigma}(t) H^{\prime}(t) \\
& -\varepsilon \frac{k_{1}^{1-p^{-}}}{p^{-}} H^{\sigma\left(p^{+}-1\right)}(t) \int_{\Omega}|u|^{p(x)} d x
\end{aligned}
$$

By using (22) and (25), we get

$$
\begin{aligned}
H^{\sigma\left(p^{+}-1\right)}(t) \int_{\Omega}|u|^{p(x)} d x & \leq H^{\sigma\left(p^{+}-1\right)}(t)\left[\int_{\Omega_{-}}|u|^{p^{-}} d x+\int_{\Omega_{+}}|u|^{p^{+}} d x\right] \\
& \leq H^{\sigma\left(p^{+}-1\right)}(t) c\left[\left(\int_{\Omega_{-}}|u|^{q^{-}} d x\right)^{\frac{p^{-}}{q^{-}}}+\left(\int_{\Omega_{+}}|u|^{q^{-}} d x\right)^{\frac{p^{+}}{q^{-}}}\right] \\
& =H^{\sigma\left(p^{+}-1\right)}(t) c\left[\|u\|_{q^{-}}^{p^{-}}+\|u\|_{q^{-}}^{p^{+}}\right] \\
& \leq c\left(\frac{1}{q^{-}} \rho_{q(.)}(u)\right)^{\sigma\left(p^{+}-1\right)}\left[\left(\rho_{q(.)}(u)\right)^{\frac{p^{-}}{q^{-}}}+\left(\rho_{q(.)}(u)\right)^{\frac{p^{+}}{q^{-}}}\right] \\
& =c_{1}\left[\left(\rho_{q(.)}(u)\right)^{\frac{p^{-}}{q^{-}}+\sigma\left(p^{+}-1\right)}+\left(\rho_{q(.)}(u)\right)^{\frac{p^{+}}{q^{-}}+\sigma\left(p^{+}-1\right)}\right]
\end{aligned}
$$


where $\Omega_{-}=\{x \in \Omega:|u|<1\}$ and $\Omega_{+}=\{x \in \Omega:|u| \geq 1\}$.

We then use Lemma 6 and (27), for

$$
s=p^{-}+\sigma q^{-}\left(p^{+}-1\right) \leq q^{-}
$$

and

$$
s=p^{+}+\sigma q^{-}\left(p^{+}-1\right) \leq q^{-},
$$

to deduce, from (35),

$$
H^{\sigma\left(p^{+}-1\right)}(t) \int_{\Omega}|u|^{p(x)} d x \leq c_{1}\left[\|\triangle u\|^{2}+\rho_{q(.)}(u)\right] .
$$

Thus, inserting estimate (36) into (34), we have

$$
\begin{aligned}
\Psi^{\prime}(t) \geq & \varepsilon\left(\beta-\frac{k_{1}^{1-p^{-}}}{p^{-}} c_{1}\right)\left[H(t)+\left\|u_{t}\right\|^{2}+\|\Delta u\|^{2}+\rho_{q(.)}(u)\right] \\
& +\left[\left(1-\sigma-\varepsilon k_{2}\right)-\varepsilon\left(\frac{p^{+}-1}{p^{+}}\right) k_{1}\right] H^{-\sigma}(t) H^{\prime}(t)
\end{aligned}
$$

Let us choose $k_{1}$ large enough so that

$$
\gamma=\beta-\frac{k_{1}^{1-p^{-}}}{p^{-}} c_{1}>0
$$

and picking $\varepsilon$ small enough such that

$$
\left(1-\sigma-\varepsilon k_{2}\right)-\varepsilon\left(\frac{p^{+}-1}{p^{+}}\right) k_{1}>0
$$

and

$$
\Psi(t) \geq \Psi(0)=H^{1-\sigma}(0)+\varepsilon \int_{\Omega} u_{0} u_{1} d x+\frac{\varepsilon}{2}\left\|\nabla u_{0}\right\|^{2}>0, \forall t \geq 0 .
$$

Consequently, (37) yields

$$
\begin{aligned}
\Psi^{\prime}(t) & \geq \varepsilon \gamma\left[H(t)+\left\|u_{t}\right\|^{2}+\|\Delta u\|^{2}+\rho_{q(.)}(u)\right] \\
& \geq \varepsilon \gamma\left[H(t)+\left\|u_{t}\right\|^{2}+\|\Delta u\|^{2}+\|u\|_{q^{-}}^{q^{-}}\right]
\end{aligned}
$$


due to (22). Therefore we get

$$
\Psi(t) \geq \Psi(0)>0, \text { for all } t \geq 0 .
$$

On the other hand, applying Hölder inequality, we obtain

$$
\begin{aligned}
\left|\int_{\Omega} u u_{t} d x\right|^{\frac{1}{1-\sigma}} & \leq\|u\|^{\frac{1}{1-\sigma}}\left\|u_{t}\right\|^{\frac{1}{1-\sigma}} \\
& \leq C\left(\|u\|_{q^{-}}^{\frac{1}{1-\sigma}}\left\|u_{t}\right\|^{\frac{1}{1-\sigma}}\right) .
\end{aligned}
$$

Young inequality gives

$$
\left|\int_{\Omega} u u_{t} d x\right|^{\frac{1}{1-\sigma}} \leq C\left(\|u\|_{q^{-}}^{\frac{\mu}{1-\sigma}}+\left\|u_{t}\right\|^{\frac{\theta}{1-\sigma}}\right)
$$

for $\frac{1}{\mu}+\frac{1}{\theta}=1$. We take $\theta=2(1-\sigma)$, to obtain $\frac{\mu}{1-\sigma}=\frac{2}{1-2 \sigma} \leq q^{-}$by (27). Therefore, (40) becomes

$$
\left|\int_{\Omega} u u_{t} d x\right|^{\frac{1}{1-\sigma}} \leq C\left(\left\|u_{t}\right\|^{2}+\|u\|_{q^{-}}^{s}\right),
$$

where $\frac{2}{1-2 \sigma} \leq q^{-}$. By using (21), we get

$$
\left|\int_{\Omega} u u_{t} d x\right|^{\frac{1}{1-\sigma}} \leq C\left(\left\|u_{t}\right\|^{2}+\|u\|_{q^{-}}^{q^{-}}+H(t)\right) .
$$

Thus, using exploiting the inequality

$$
\left(a_{1}+a_{2}+\ldots+a_{m}\right)^{\lambda} \leq 2^{(m-1) /(\lambda-1)}\left(a_{1}^{\lambda}+a_{2}^{\lambda}+\ldots+a_{m}^{\lambda}\right),
$$

$\left(a_{1}, a_{2}, \ldots, a_{m} \geq 0, \lambda \geq 1\right)$, we have

$$
\begin{aligned}
\Psi^{\frac{1}{1-\sigma}}(t) & =\left[H^{1-\sigma}(t)+\varepsilon \int_{\Omega} u u_{t} d x+\frac{\varepsilon}{2}\|\nabla u\|^{2}\right]^{\frac{1}{1-\sigma}} \\
& \leq 2^{\frac{\sigma}{1-\sigma}}\left(H(t)+\varepsilon^{\frac{1}{1-\sigma}}\left|\int_{\Omega} u u_{t} d x\right|^{\frac{1}{1-\sigma}}\right) \\
& \leq C\left(\left\|u_{t}\right\|^{2}+\|u\|_{q^{-}}^{q^{-}}+H(t)\right) \\
& \leq C\left(H(t)+\left\|u_{t}\right\|^{2}+\|\triangle u\|^{2}+\|u\|_{q^{-}}^{q^{-}}\right) .
\end{aligned}
$$

By combining of (39) and (41), we arrive

$$
\Psi^{\prime}(t) \geq \xi \Psi^{\frac{1}{1-\sigma}}(t)
$$


where $\xi$ is a positive constant.

A simple integration of $(42)$ over $(0, t)$ yields $\Psi^{\frac{\sigma}{1-\sigma}}(t) \geq \frac{1}{\Psi^{-\frac{\sigma}{1-\sigma}}(0)-\frac{\xi \sigma t}{1-\sigma}}$, which implies that the solution blows up in a finite time $T^{*}$, with

$$
T^{*} \leq \frac{1-\sigma}{\xi \sigma \Psi^{\frac{\sigma}{1-\sigma}}(0)}
$$

This completes the proof of the theorem.

\section{Conflict of interest}

The authors declare no potential conflict of interests. 


\section{References}

(1989).

(1994).

(1989).

(2003).

(327AD).

(1994).

(1995).

(2008).

(2006).

(2012).

(2014).

(2011).

(2009).

(1147).

(2020).

(1996).

(2008).

(2012). 